\title{
Cell-free long non-coding RNA-H19 and miRNA-29a, miRNA-29b expression in Type 2 Diabetes Mellitus patients.
}

Mohammed Alfaifi

King Khalid University

Amit Kumar Verma

Gurukula Kangri Vishwavidyalaya

Mohammed Yahya Alshahrani

King Khalid University

Prakash C Joshi

Gurukula Kangri Vishwavidyalaya

Ali Gaithan Alkhathami

King Khalid University

Irfan Ahmad

King Khalid University

Abdulrahim Refdan Hakami

King Khalid University

Mirza Masroor Ali Beg (D mirzamasroor1986@gmail.com )

Jamia Hamdard https://orcid.org/0000-0002-4519-721X

Research article

Keywords: Diabetes, IncRNA, microRNA, Gene expression, Cell-free system.

Posted Date: August 28th, 2020

DOI: https://doi.org/10.21203/rs.3.rs-47940/v1

License: (c) (i) This work is licensed under a Creative Commons Attribution 4.0 International License. Read Full License 


\section{Abstract}

Background: Type 2 diabetes mellitus [T2DM] has been one of the common diseases and characterized by increased blood glucose levels. T2DM participates in several organ damages as well revealed that cellfree non-coding RNAs and microRNAs (miRNAs) have been demonstrated to serve as important diagnostic/prognostic biomarkers in the pathophysiology of diabetes.

Materials/Methods: The present study included clinically confirmed untreated newly diagnosed 200 cases of T2DM and 200 healthy subjects. Blood samples collected in fluoride vials were used to check blood glucose for fasting and after 2 hours of breakfast (postprandial), blood samples collected in EDTA vials used for the Hba1c level.

Blood samples from all the participants were collected in plain vials used for cell-free total RNA extraction. Total extracted RNA was quantified and 100ng was used to synthesized the cDNA for cell-free IncRNA H19, miRNA-29a, and miRNA-29b expression using specific primers/ probes using quantitative real-time PCR method. Serum Biochemical parameters were analyzed after collection of the sample to observe the changes among T2DM cases and healthy controls. Subjective data were recorded to evaluate the association with IncRNA H19, miRNA-29a, and miRNA-29b expression among T2DM cases.

Results: It was observed that type 2 diabetic patients had decreased [0.59 fold] IncRNA H19 expression while increased miRNA-29a [5.62 fold] and miRNA-29b [5.58 fold] expression. Decreased expression of IncRNA H19 was observed to be associated with gender [p=0.004], hypertension [ $<<0.0001]$, weight loss $[p=0.02]$ and fatigue $[p=0.02]$. Increased miRNA29a expression was linked with hypertension $[p<0.0001]$, alcoholism [ $p=0.04]$, and smoking $[p<0.0001]$ as well as miRNA-29b expression was associated with hypertension [ $p=0.0001]$, weight loss [ $p=0.002]$, smoking $[p=0.0002]$, alcoholism $[p<0.0001]$. Low $[<1$ fold] and high [ $>1$ fold] expression of IncRNA H19 expression was linked with miRNA-29a [p=0.005] and miRNA-29b [ $<0.0001]$ expression. IncRNA H19 expression showed negative correlation with miRNA-29a expression $[r=-27, p<0.0001]$ and miRNA-29b $[r=-47, p<0.0001]$.

Conclusion: The present study concluded that lower IncRNA H19 expression, and increased miRNA-29b, miRNA-29b expression associated with the severity of T2DM patients. Decreased IncRNA H19 expression, and increased miRNA-29b, miRNA-29b expression observed to be interrelated with clinicopathological findings of T2DM patients could involve in pathogenesis disease.

\section{Introduction}

Type 2 diabetes mellitus [T2DM] is strongly associated with impaired glucose level, which developed due to the inability of the pancreas to secret enough insulin as well as insulin resistance. Hyperglycemia causes injury to major organs such as blood vessels, liver, brain, heart, kidneys, eyes, and can heavily contribute to the health and financial burden associated with the disease. The liver is particularly involved in maintaining blood glucose levels and excessive hepatic glucose production [HGP] contributing hyperglycemia in T2DM patients [1, 2]. Numerous long non-coding RNAs [IncRNAs] have been identified, 
and projects like ENCODE have focused on the identification and characterization of IncRNAs. ENCODE project revealed that there are at least 9,640 human loci IncRNAs, and the number grows continuously [3]. LncRNAs play a significant role in regulation at transcriptional and, post-transcriptional levels, and the organization of chromatin [4]. Studies suggested that LncRNAs also play a vital role in tumorigenesis [5]. LncRNAs and microRNAs [miRNAs] have different functions and participate in translational repression by targeting the genes, thereby shifting the nature of disease states. LncRNAs have involved in multiple cellular processes and emerged as potent genetic regulators. LncRNAs serve as a negative regulator of miRNAs and contributes to inhibits the level of expression [6]. Inc RNA H19 is positioned on chromosome number 11 p15.5, about $100 \mathrm{~kb}$ distal of insulin-like growth factor 2 [IGF2], and simultaneously H19 and IGF2 are transcribed from a conserved embossed gene cluster [7]. In T2DM patients, approximately 5 times lower $\mathrm{H} 19$ levels were observed in skeletal muscle compared to healthy individuals [8]. Considerable attention received on multiple genes linked to epigenetic regulation of path-physiology of diabetes. MiRNAs are a group of short sequence about 22 nucleotides and target the multiple mRNAs that regulate protein-coding genes expression [9] and have been predicted that miRNAs regulate over $60 \%$ of all coding genes in mammals [10]. Several miRNAs have been associated with impairment of glycemic homeostasis and the diabetes progression [11, 12].

The human miRNA-29 family abides miRNA-29a, miRNA-29b, and located and transcribed at chromosome 7 [7q32.3], whereas miRNA-29c is transcribed from chromosome 1 [1q32.2] [13]. Clusters of miRNA-29 transcribed together as polycistronic primary transcripts [14]. Increased miRNA29 family was observed in diabetic rodent models tissues such as liver, $\beta$-cells, kidney cells, skeletal muscle cells, and adipose tissue [14].

IncRNA H19 could bind to miRNA-29a competitively following miRNA response elements (MREs) of miRNA-29a [15]. Zhong et al revealed that that IncH19 sponge the miRNA-29b and affect its expression [16] and Mengxin Lv et al also have been stated that $\mathrm{H} 19$ could directly bind to miR-29b reduces its expression [17]. Therefore, present research work aimed to investigate the clinical relevance of cell-free IncRNA H19, miRNA-29a, miRNA-29b expression level, and their association with clinical features/variables in T2DM patients in Indian population.

\section{Materials And Methods}

\section{Sample collection}

This study included a total of 400 study subjects, 200 were newly diagnosed untreated type 2 diabetes mellitus patients and, 200 were healthy subjects. $3 \mathrm{ml}$ of blood samples were withdrawn from all the study subjects and collected in plain vials. Fasting as well as postprandial samples collected in fluoride vials from all the 200 T2DM subjects and $1 \mathrm{ml}$ blood in EDTA vials for Hba1c. All the criteria were followed, such as fasting blood glucose [glucose level $\geq 126 \mathrm{mg} / \mathrm{dL}$ ] and postprandial glucose [2-hour blood glucose $\geq 200 \mathrm{mg} / \mathrm{dL}$ ] were monitored for diagnosis of T2DM disease. T2DM patients with Hypertension ( $\mathrm{BP} \geq 140$ or $\geq 90 \mathrm{~mm} \mathrm{Hg}$ ), increased urination (polyurea by physical examination as well 
as $8,12 \& 24 \mathrm{hrs}$ urine test), weight loss ( $>4 \mathrm{~kg}$ weight loosed in last 3 to 6 months), fatigue, wound healing, blur vision (Fundus/ fluorescein angiography), loss of appetite, smoking, alcoholism were subjective data recorded for analysis.

Blood samples centrifuged at $1500 \mathrm{rpm}$ to collect the serum and stored at $-80^{\circ} \mathrm{C}$ for further process. The ethical committee board ethically approved this research study and informed written consent was obtained from all the participants before the study commenced. The present study was ethically approved and the study was conducted at the Department of Zoology, Gurukula Kangri University, Haridwar, India.

\section{Total RNA extraction}

Stored serum samples of T2DM cases and healthy controls were thawed, and total cell-free RNA extraction was done by kit [mirVana total RNA isolation kit, Thermo Fisher Scientific]. All samples are then stored at $-70^{\circ} \mathrm{C}$ in $2 \mathrm{ml}$ nuclease-free Eppendorf tubes. The concentration and quality of cell-free RNA were checked by the A260/280 ratio using Nano-drop.

\section{cDNA synthesis and QRT-PCR for IncH19 expression study}

$100 \mathrm{ng}$ of the total cell-free extracted RNA from T2DM patients and healthy controls were used to synthesized cDNA using kit [Verso, Thermo scientific, USA] following kit provided protocol. Expression of cell-free IncRNA H19 was done by quantitative real-time PCR using SYBR green dye using forward primer sequence 5'-ATCGGTGCCTCAGCGTTCGG-3' and reverse primer sequence 5'-CTGTCCTCGCCGTCACACCG$3^{\prime}$ and $\beta$-actin were used as internal control, and the forward primer sequence 5 'CGACAACGGCTCCGGCATGTGC-3', reverse primer sequence 5'-GTCACCGGAGTCCATCACGATGC-3'. The program followed as qRT-PCR for IncRNA H19 and $\beta$-actin was performed for 40 cycles; initial denaturation was at $94^{\circ} \mathrm{C}$ for 40 seconds, annealing temperature was at $60^{\circ} \mathrm{C}$ for 40 seconds, extension at $72^{\circ} \mathrm{C}$ for 40 seconds and 20 micro litters reaction volume was used. Ending additional step at $72^{\circ} \mathrm{C}$ for 5 minutes to end up reaction and melting curve examined between the ranges $35^{\circ} \mathrm{C}$ to $90^{\circ} \mathrm{C}$ to confirm the target amplification. A control without cDNA was included in each experiment, and every reaction was done in duplicate. The relative quantification by $2^{-[\Delta C T]}$ method was used to compute the IncRNA H19 level expression.

\section{Polyadenylation, cDNA synthesis for miRNA-29a, and miRNA-29b expression study}

Total 100 ng RNA was taken to polyadenylate and cDNA synthesis by miRNA Reverse transcription kit [TaqMan, Thermo Fisher Scientific] by following kit protocol. Essential reagents such as reverse transcriptase enzyme and other reagents were added to switch in poly $[A]$ - tail and then into cDNA using a universal RT primer provided with the kit.

\section{QRT-PCR for miRNA-29a, and miRNA-29b expression}


Quantitative real-time PCR [qPCR] was performed to compute the expression level of cell-free miRNA-29a and miRNA-29b. qPCR was done by advanced Taqman master mix [4444556], Taqman probes for miRNA-29a [478587_mir], and miRNA-29b [478369_mir] for quantification and miRNA-16 [477860_mir] were used as internal control as normalizer to calculate the level of expression.

\section{Statistical analysis:}

All the statistical testing was done using Graph Pad Prism software version 6.05 and SPSS 20.0 version. Mann Whitney $U$ test used to calculate the differences in biochemical parameters among T2DM subjects and healthy controls. QRT-PCR outcome analysis was done by the relative cycle threshold [Ct] method to compute the LncRNA H19, miRNA-29a, and miRNA-29b expression levels by the relative quantification method using the $2^{-[\Delta \triangle C t]}$. The Mann - Whitney $U$ test was done to calculate the differences in IncRNA H19, miRNA29a, miRNA29b expression level among the subjective variables. Results more than or less than one were considered to specify up-regulation or down-regulation of expression.

Spearman correlation analysis was performed to check the degree of association by calculating the rvalue of IncRNA H19 with miRNA-29a and miRNA-29b. Regression analysis was performed to calculate the $\mathrm{R}^{2}$ and beta coefficient to check the effect of IncRNA H19 and biochemical parameters on miRNA-29a and miRNA-29b expression.

All values were normalized relative to the control values, which were depicted as a value of 1 . P-value of less than 0.05 was considered statistically significant.

\section{Results}

\section{Demographic and clinical characteristics of T2DM patients and healthy controls}

In brief, the present study included 200 cases of type 2 diabetes mellitus and 200 healthy control subjects [Table 1]. Among the T2DM cases, $68 \%$ of patients were males, and $32 \%$ were females while in healthy controls, $70 \%$ were males, and $30 \%$ were females, more details mentioned in Table 1 . 
Table 1

Demographic and clinical characteristics of enrolled patients with Type 2 diabetes mellitus and healthy controls.

\begin{tabular}{|c|c|c|}
\hline Variables & T2DM patients (\%) & Controls subjects (\%) \\
\hline Total no. & $200(100)$ & $200(100)$ \\
\hline \multicolumn{3}{|l|}{ Gender } \\
\hline Males & $136(68)$ & $140(70)$ \\
\hline Females & $64(32$ & $60(30)$ \\
\hline \multicolumn{3}{|c|}{ Age at diagnosis (Years) } \\
\hline$\leq 40$ & $94(47)$ & $100(50)$ \\
\hline$>40$ & $106(53)$ & $100(50)$ \\
\hline Mean age (in years) & $46.32 \pm 7.18$ & $47.63 \pm 6.76$ \\
\hline \multicolumn{3}{|l|}{ Hypertension } \\
\hline Yes & $122(61)$ & \\
\hline No & $78(39)$ & \\
\hline \multicolumn{3}{|l|}{ Increased urination } \\
\hline Yes & $97(48.5)$ & \\
\hline No & $103(51.5)$ & \\
\hline \multicolumn{3}{|l|}{ Weight loss } \\
\hline Yes & $94(47)$ & \\
\hline No & $106(53)$ & \\
\hline \multicolumn{3}{|l|}{ Fatigue } \\
\hline Yes & $95(47.5)$ & \\
\hline No & $105(52.5)$ & \\
\hline \multicolumn{3}{|l|}{ Wound healing } \\
\hline Yes & $84(42)$ & \\
\hline No & $116(58)$ & \\
\hline \multicolumn{3}{|l|}{ Blur vision } \\
\hline Yes & $67(33.5)$ & \\
\hline No & $133(76.5)$ & \\
\hline
\end{tabular}




\begin{tabular}{|ll|}
\hline Variables & T2DM patients (\%) \\
\hline Loss of appetite & \\
\hline Yes & $91(45.5)$ \\
\hline No & $109(54.5)$ \\
\hline Smoking status & \\
\hline Yes & $92(46)$ \\
\hline No & $108(54)$ \\
\hline Alcoholism & \\
\hline Yes & $102(51)$ \\
\hline No & $98(49)$ \\
\hline
\end{tabular}

\section{Association of anthropometric data and biochemical parameters among T2DM and healthy subjects}

Study recorded anthropometric data and biochemical data and depicted in Table 2. In T2DM, Hip, waist, and body mass index (BMI) were $35.97,32.61$, and 29.0 while in healthy subjects, $34.87,31.65$, and 24.82 and differences among them were observed to be statistically significant $(p=0.004, p=0.02, p<0.0001)$ respectively. Blood glucose parameters such as fasting, postprandial, and $\mathrm{Hba} 1 \mathrm{c}$ were tested and differences among T2DM and healthy subjects were observed to be significant $(p<0.0001, p<0.0001, p$ $<0.0001)$.

Systolic and diastolic blood presser differences among T2DM and healthy subjects were statistically significant $(p<0.0001, p=0.0007)$. Lipid profile analysis such as High-density lipoprotein $(H D L)$, Lowdensity lipoprotein (LDL), Triglycerides (TG), Very low-density lipoprotein (VLDL), cholesterol showed significant differences among T2DM and healthy control subjects $(p<0.0001, p<0.0001, p<0.0001, p<$ $0.0001, p<0.0001)$ respectively. 
Table 2

Comparison of anthropometric data and biochemical parameters among T2DM and healthy subjects.

\begin{tabular}{|llll|}
\hline Parameters & $\begin{array}{l}\text { T2DM cases } \\
\text { (Mean } \pm \text { SD) }\end{array}$ & $\begin{array}{l}\text { Healthy control } \\
\text { (Mean } \pm \text { SD) }\end{array}$ & p value \\
\hline Age (years) & $46.32 \pm 7.18$ & $47.63 \pm 6.76$ & 0.13 \\
\hline Hip (cm) & $35.97 \pm 3.59$ & $34.87 \pm 1.87$ & 0.004 \\
\hline Waist (cm) & $32.61 \pm 3.84$ & $31.65 \pm 2.50$ & 0.02 \\
\hline Weight (kg) & $71.64 \pm 13.63$ & $70.74 \pm 7.79$ & 0.53 \\
\hline Body mass index & $29.0 \pm 5.51$ & $24.82 \pm 2.33$ & $<0.0001$ \\
\hline Fasting blood glucose & $240.0 \pm 18.8$ & $90.22 \pm 7.10$ & $<0.0001$ \\
\hline Postprandial blood glucose & $349.0 \pm 75$ & $136.0 \pm 5.0$ & $<0.0001$ \\
\hline Hba1c & $7.39 \pm 0.77$ & $5.22 \pm 0.58$ & $<0.0001$ \\
\hline Blood pressure (systolic) & $129.4 \pm 13.99$ & $118.32 \pm 10.78$ & $<0.0001$ \\
\hline Blood pressure (diastolic) & $86.76 \pm 13.31$ & $81.90 \pm 6.18$ & 0.0007 \\
\hline High density lipoprotein (HDL) & $42.42 \pm 12.9$ & $49.78 \pm 6.92$ & $<0.0001$ \\
\hline Low density lipoprotein (LDL) & $192.2 \pm 27.0$ & $124.54 \pm 14.20$ & $<0.0001$ \\
\hline Triglycerides (TG) & $375.4 \pm 117.7$ & $140.98 \pm 5.52$ & $<0.0001$ \\
\hline Very low density lipoprotein (VLDL) & $35.92 \pm 5.18$ & $26.52 \pm 2.53$ & $<0.0001$ \\
\hline Cholesterol & $248.5 \pm 13.45$ & $152.63 \pm 18.82$ & $<0.0001$ \\
\hline
\end{tabular}

\section{LncRNA H19 expression in T2DM patients}

It was found that T2DM patients had an overall 0.59-fold expression of IncRNA H19 compared to healthy controls [Table 3]. Among the T2DM patients, a significant difference in IncRNA H19 expression was observed between males and females [P $=0.004]$. Males showed 0.70 -fold IncRNA H19 expression while females had 0.56-fold IncRNA H19 expression. Hypertensive T2DM patients, had 0.41 fold changes in IncRNA H19 expression, while non-hypertensive patients showed 0.88 fold IncRNA H19 expression, and statistical differences between them were observed to be significant $[P<0.0001]$. T2DM patients with weight-loss showed 0.41 fold IncRNA H19 expression, while patients without weight loss showed 0.79 fold IncRNA H19 expression [P = 0.02]. Besides, T2DM patients who had fatigue showed 0.47 fold IncRNA H19 expression, while patients with no fatigue showed 0.73 fold IncRNA H19 expression [P = 0.02]. 
Table 3

Long non-coding RNA H19 expression in type 2 Diabetes mellitus subjects.

\begin{tabular}{|c|c|c|}
\hline Variables & H19 expression [Mean \pm SD] & P-value \\
\hline Overall expression & $0.59 \pm 0.89$ & - \\
\hline \multicolumn{3}{|l|}{ Age [in years] } \\
\hline$\leq 40$ years & $0.70 \pm 1.07$ & \multirow[t]{2}{*}{0.82} \\
\hline$>40$ years & $0.56 \pm 0.82$ & \\
\hline \multicolumn{3}{|l|}{ Gender } \\
\hline Male & $0.41 \pm 0.68$ & \multirow[t]{2}{*}{0.004} \\
\hline Female & $0.81 \pm 1.04$ & \\
\hline \multicolumn{3}{|l|}{ Hypertension } \\
\hline Yes & $0.41 \pm 0.85$ & \multirow[t]{2}{*}{$<0.0001$} \\
\hline No & $0.88 \pm 0.87$ & \\
\hline \multicolumn{3}{|l|}{ Urination } \\
\hline Yes & $0.62 \pm 0.92$ & \multirow[t]{2}{*}{0.46} \\
\hline No & $0.56 \pm 0.82$ & \\
\hline \multicolumn{3}{|l|}{ Weight loss } \\
\hline Yes & $0.41 \pm 0.74$ & \multirow[t]{2}{*}{0.02} \\
\hline No & $0.79 \pm 0.99$ & \\
\hline \multicolumn{3}{|l|}{ Fatigue } \\
\hline Yes & $0.47 \pm 0.78$ & \multirow[t]{2}{*}{0.02} \\
\hline No & $0.73 \pm 0.98$ & \\
\hline \multicolumn{3}{|l|}{ Wound healing } \\
\hline Yes & $0.59 \pm 0.86$ & \multirow[t]{2}{*}{0.68} \\
\hline No & $0.59 \pm 0.91$ & \\
\hline \multicolumn{3}{|l|}{ Blur vision } \\
\hline Yes & $0.50 \pm 0.74$ & \multirow[t]{2}{*}{0.87} \\
\hline No & $0.64 \pm 0.95$ & \\
\hline
\end{tabular}




\begin{tabular}{|lll|}
\hline Variables & H19 expression [Mean \pm SD] & P-value \\
\hline Yes & $0.55 \pm 0.83$ & 0.41 \\
\hline No & $0.64 \pm 0.93$ & \\
\hline Smoking & & 0.13 \\
Yes & $0.49 \pm 0.78$ & \\
No & $0.68 \pm 0.96$ & 0.52 \\
\hline Alcoholism & & \\
\hline Yes & $0.48 \pm 0.75$ & \\
\hline No & $0.70 \pm 1.0$ & \\
\hline
\end{tabular}

\section{Association of microRNA-29a among T2DM patients}

T2DM patients showed an overall 5.62 mean fold expression of miRNA-29a compared to healthy controls [Table 4]. T2DM patients who were hypertensive showed 5.96-fold miRNA-29a expression, whereas nonhypertensive reported having 5.09-fold miRNA-29a expression. Besides, the differences between diabetic smoker and non-smoker patients in $\mathrm{H} 19$ expression were observed to be statistically significant $[\mathrm{P}<$ 0.0001]. We also studied whether cigarette smoking has an impact on miRNA-29a expression. We observed that the T2DM patients with smoking habit showed 5.98-fold miRNA-29a expression, and nonsmokers showed 5.24-fold miRNA-29a expression [P=0.04]. T2DM subjects with the habit of alcohol consumption showed 6.17-fold miRNA-29a expression, while non-alcoholic T2DM patients showed 5.04fold miRNA-29a expression. Furthermore, a statistically significant correlation was observed in $\mathrm{H} 19$ expression between alcoholic and non-alcoholic diabetic patients $[\mathrm{P}<0.0001]$. Our observations illustrated that miRNA-29a expression in T2DM patients is directly associated with hypertension and alcohol consumptions. 
Table 4

miR-29a expression in subjects with Type 2 Diabetes Mellitus.

\begin{tabular}{|c|c|c|}
\hline Variables & miRNA-29a expression [Mean \pm SD] & P-value \\
\hline Overall expression & $5.62 \pm 1.92$ & - \\
\hline \multicolumn{3}{|l|}{ Age [in years] } \\
\hline$\leq 40$ years & $5.57 \pm 1.55$ & \multirow[t]{2}{*}{0.64} \\
\hline$>40$ years & $5.63 \pm 2.03$ & \\
\hline \multicolumn{3}{|l|}{ Gender } \\
\hline Male & $5.69 \pm 1.84$ & \multirow[t]{2}{*}{0.14} \\
\hline Female & $5.53 \pm 2.03$ & \\
\hline \multicolumn{3}{|l|}{ Hypertension } \\
\hline Yes & $5.96 \pm 1.84$ & \multirow[t]{2}{*}{$<0.0001$} \\
\hline No & $5.09 \pm 1.95$ & \\
\hline \multicolumn{3}{|l|}{ Urination } \\
\hline Yes & $5.58 \pm 1.97$ & \multirow[t]{2}{*}{0.64} \\
\hline No & $5.65 \pm 1.89$ & \\
\hline \multicolumn{3}{|l|}{ Weight loss } \\
\hline Yes & $5.81 \pm 2.14$ & \multirow[t]{2}{*}{0.21} \\
\hline No & $5.40 \pm 1.63$ & \\
\hline \multicolumn{3}{|l|}{ Fatigue } \\
\hline Yes & $5.62 \pm 1.92$ & \multirow[t]{2}{*}{0.88} \\
\hline No & $5.62 \pm 1.93$ & \\
\hline \multicolumn{3}{|l|}{ Wound healing } \\
\hline Yes & $5.67 \pm 1.95$ & \multirow[t]{2}{*}{0.70} \\
\hline No & $5.57 \pm 1.92$ & \\
\hline \multicolumn{3}{|l|}{ Blur vision } \\
\hline Yes & $5.68 \pm 2.0$ & \multirow[t]{2}{*}{0.65} \\
\hline No & $5.49 \pm 1.78$ & \\
\hline \multicolumn{3}{|l|}{ Loss of appetite } \\
\hline Yes & $5.74 \pm 2.15$ & 0.75 \\
\hline
\end{tabular}




\begin{tabular}{|lll|}
\hline Variables & miRNA-29a expression [Mean \pm SD] & P-value \\
\hline No & $5.52 \pm 1.72$ & \\
\hline Smoking & & \\
\hline Yes & $5.98 \pm 1.90$ & \\
\hline No & $5.24 \pm 1.58$ & \\
Alcoholism & & $<0.0001$ \\
Yes & $6.17 \pm 2.09$ & \\
\hline No & $5.04 \pm 1.55$ & \\
\hline
\end{tabular}

\section{Association of microRNA-29b expression among T2DM patients}

T2DM patients showed an overall 5.58 mean fold expression of miRNA-29b compared to healthy controls [Table 5]. T2DM patients who were hypertensive showed 5.85-fold miRNA-29b expression while nonhypertensive showed 5.17-fold miRNA-29b expression. Statistical analyses revealed differences between analyzed groups $[P=0.0001]$. Furthermore, T2DM patients who had weight-loss showed 5.91-fold miRNA$29 \mathrm{~b}$ expression, while those who did not show weight loss had 5.21 -fold miRNA-29b expression $[\mathrm{P}=$ 0.002]. Smokers T2DM patients showed 6.16-fold miRNA-29b expression, while non-smokers showed 5.15-fold miRNA-29b expression [P = 0.0002]. Alcoholic T2DM patients showed 6.11-fold miRNA-29b expression, while non-alcoholic T2DM patients showed 5.05-fold miRNA-29b expression [P $<0.0001]$. 
Table 5

miR-29b expression in Type 2 Diabetes Mellitus patients.

\begin{tabular}{|c|c|c|}
\hline Variables & miR-29b expression [Mean \pm SD] & P-value \\
\hline Overall expression & $5.58 \pm 1.83$ & - \\
\hline \multicolumn{3}{|l|}{ Age [in years] } \\
\hline$\leq 40$ years & $5.39 \pm 1.73$ & \multirow[t]{2}{*}{0.41} \\
\hline$>40$ years & $5.65 \pm 1.86$ & \\
\hline \multicolumn{3}{|l|}{ Gender } \\
\hline Male & $5.41 \pm 1.79$ & \multirow[t]{2}{*}{0.25} \\
\hline Female & $5.69 \pm 1.97$ & \\
\hline \multicolumn{3}{|l|}{ Hypertension } \\
\hline Yes & $5.85 \pm 1.68$ & \multirow[t]{2}{*}{0.0001} \\
\hline No & $5.17 \pm 1.97$ & \\
\hline \multicolumn{3}{|l|}{ Urination } \\
\hline Yes & $5.71 \pm 2.03$ & \multirow[t]{2}{*}{0.80} \\
\hline No & $5.47 \pm 1.62$ & \\
\hline \multicolumn{3}{|l|}{ Weight loss } \\
\hline Yes & $5.91 \pm 1.86$ & \multirow[t]{2}{*}{0.002} \\
\hline No & $5.21 \pm 1.72$ & \\
\hline \multicolumn{3}{|l|}{ Fatigue } \\
\hline Yes & $5.47 \pm 1.87$ & \multirow[t]{2}{*}{0.20} \\
\hline No & $5.69 \pm 1.79$ & \\
\hline \multicolumn{3}{|l|}{ Wound healing } \\
\hline Yes & $5.69 \pm 1.84$ & \multirow[t]{2}{*}{0.41} \\
\hline No & $5.51 \pm 1.82$ & \\
\hline \multicolumn{3}{|l|}{ Blur vision } \\
\hline Yes & $6.01 \pm 2.13$ & \multirow[t]{2}{*}{0.09} \\
\hline No & $5.37 \pm 1.62$ & \\
\hline \multicolumn{3}{|l|}{ Loss of appetite } \\
\hline Yes & $5.58 \pm 1.79$ & 0.97 \\
\hline
\end{tabular}




\begin{tabular}{|lll|}
\hline Variables & miR-29b expression [Mean \pm SD] & P-value \\
\hline No & $5.59 \pm 1.87$ & \\
\hline Smoking & & 0.0002 \\
\hline Yes & $6.16 \pm 2.07$ & \\
\hline No & $5.10 \pm 1.43$ & $<0.0001$ \\
Alcoholism & & \\
\hline Yes & $6.11 \pm 2.04$ & \\
\hline No & $5.04 \pm 1.39$ & \\
\hline
\end{tabular}

\section{Relationship of biochemical parameters with IncRNA H19, miRNA-29a, and miRNA-29b expression among T2DM patients}

Regression analysis was done to check the effect of all the parameters such as H19, VLDL, PPG, FPG, BP (Diastolic), Cholesterol, BP (systolic), Hba1c, HDL, TG, LDL with miRNA29a, miRNA29b expression, and the observed $R^{2}$ value was $0.13(p=0.003)$ and $0.14(p=0.001)$ (Table 6$)$. The beta coefficient was also calculated and the observed beta value for IncH19 to miRNA-29a was $-0.298(p<0.0001)$ and concerning miRNA-29a was-0.028 $(p<0.0001)$ (Table 7).

Table 6

Association of IncRNA H19 with miRNA29a and miRNA29b.

\begin{tabular}{|llc|}
\hline Parameters & $\mathbf{R}^{2}$ & P-value \\
\hline Dependent Variable: miRNA29a & 0.13 & .003 \\
\hline Dependent Variable: miRNA29b & 0.14 & .001 \\
\hline $\begin{array}{l}\text { Predictors: (Constant), H19, VLDL, PPG, FPG, BP (Diastolic), Cholesterol, BP (systolic), Hba1c, HDL, } \\
\text { TG, LDL. }\end{array}$ & \\
\hline
\end{tabular}


Table 7

Association of IncRNA H19 and biochemical parameters with miRNA29a and miRNA29b.

\begin{tabular}{|c|c|c|c|c|}
\hline \multirow[t]{2}{*}{ Variables } & Standardized Coefficients & \multirow[t]{2}{*}{ P-value } & Standardized Coefficients & \multirow[t]{2}{*}{ P-value } \\
\hline & Beta & & Beta & \\
\hline H19 & -0.298 & 0.0001 & -0.285 & 0.0001 \\
\hline FPG & 0.075 & 0.284 & 0.042 & 0.549 \\
\hline PPG & -0.015 & 0.848 & -0.124 & 0.124 \\
\hline Hba1c & 0.110 & 0.130 & 0.134 & 0.062 \\
\hline BP (systolic) & 0.115 & 0.111 & 0.122 & 0.090 \\
\hline BP(Diastolic) & -0.109 & 0.120 & -0.073 & 0.293 \\
\hline HDL & -0.106 & 0.189 & 0.102 & 0.202 \\
\hline LDL & -0.129 & 0.190 & 0.099 & 0.312 \\
\hline TG & 0.089 & 0.354 & 0.013 & 0.893 \\
\hline Cholesterol & -0.015 & 0.856 & -0.070 & 0.404 \\
\hline VLDL & -0.019 & 0.785 & -0.026 & 0.702 \\
\hline \multicolumn{3}{|c|}{ Dependent Variable: miRNA29a } & \multicolumn{2}{|c|}{ Dependent Variable: miRNA29b } \\
\hline
\end{tabular}

\section{Association of IncRNA H19 with miRNA-29a and miRNA-29b}

Significant association of low [ $\leq 1 \mathrm{fold}]$ and high [ $>1 \mathrm{fold}]$ expression of IncRNA H19 with miRNA29a and miRNA29b was observed [Figure 1]. It was analyzed that the T2DM patients who had low expression of IncRNA H19 showed 5.91-fold miRNA-29a expression and high expression of IncRNA H19 showed 4.60 fold changes in miRNA-29a expression and difference among them was found to be statistically significant $[P=0.005]$. T2DM patients who had low expression of IncRNA H19 showed 7.21-fold miRNA29b expression and high expression of IncRNA H19 showed 4.69-fold miRNA-29b expression was observed $[\mathrm{P}<0.0001]$.

\section{Correlation of IncRNA H19 with miRNA-29a and miRNA-29b}

A negative correlation of IncRNA H19 with miRNA-29a and miRNA-29b was observed [Figure 2]. It was found that IncRNA H19 expression showed negative correlation with miRNA-29a [ $r=-0.27, \mathrm{P}<0.0001]$ as well as negative correlation with miRNA-29b $[\mathrm{r}=-0.47, \mathrm{P}<0.0001]$ was also observed.

\section{Discussion}


Diabetes mellitus is a metabolic disorder that developed due to insufficient insulin secretion from $\beta$-cells of the pancreas [18]. The frequency of T2DM has increased in a few decades and to date affects more than three hundred million lives worldwide due to increased sedentary life and easy access to fast foods [19]. The pathogenesis of T2DM is associated with a genetic disposition, environmental exposures, and multiple causes. [20]. Long non-coding RNA H19 is a multifunctional long noncoding RNA that functionally involved in the nucleus and the cytoplasm [21]. In T2DM patients, five times lower H19 expression observed in skeletal muscle compared to healthy individuals [22]. Zhuo et al. have shown that diabetic rats had decreased expression of $\mathrm{H} 19$ as well as in high glucose exposed to neonatal cardiomyocytes [23].

A downregulation of cell-free IncRNA H19 observed in diabetes [24], and in the same way, the present study revealed decreased cell-free IncRNA H19 expression associated with gender, hypertension, weight loss, and fatigue. Male T2DM patients showed 0.40-fold down-regulation, hypertensive T2DM patients showed 0.47-fold down-regulation, T2DM patients who had weight-loss showed 0.38-fold downregulation, T2DM patients who had fatigue showed 0.26-fold down-regulation of IncRNA H19 expression compared to its counterpart. Increased cell-free miRNA-29a and miRNA-29b expression were observed among T2DM patients. It has revealed that the cell-free miRNA-29a expression was linked with hypertension and alcoholism. miRNA-29b expression was linked with hypertension, weight loss, smoking, and alcoholism. Increased expression of the miRNA-29 family recorded in different tissues such as the liver [25] and $\beta$ cells of diabetic rodent models and humans [26].

Essentially, an increase in miRNA-29 expression level in the serum of children diagnosed with T1DM and adult T2DM patients was observed [27]. Hyperglycemia is the primary situation of diabetes mellitus and leads to up-regulates the expression of miRNA-29a and miRNA-29b [28]. Roggli et al. revealed that patients treated with proinflammatory cytokine induce the miRNA-29 family members' expression level in human beings and mouse pancreatic cells. Moreover, an increase in the expression of miRNA-29 family miRNAs is associated with the early step of diabetes in mice [29]. It has been shown that miRNA-29 suppression by using anti-miRNA-29 oligomers decreases the expression and protects the patients against diabetic nephropathy [28].

Pancreatic islets showed upregulation of miRNA-29a and miRNA-29b and involved in $\beta$-cell death, initiation of diabetes, and suggested to be the reliable diabetic marker [26]. Dahlman has revealed that the T2DM patient's skeletal muscles showed increased expression of miRNA-29 family members compared to the non-diabetic person [30]. miRNA-29b was over-expressed in diabetic rat's muscle cells and correlated with the negative GLUT4 expression, and miRNA-29a has previously been validated as repressors of GLUT4, increased miRNA-29a expression was observed in vastus lateralis muscle T2DM, and GLUT4 protein expression was observed to decrease in mice skeletal muscle over-expressing miRNA29a [31], as well as in C2C12 muscle cells [32]. Expression of GLUT4 level has been reported to decrease in 3T3-L1 adipocytes over-expressing miRNA-29a, miRNA-29b [33]. 
This study observed the T2DM patients who had expression level $\leq 1$ fold of IncRNA H19 showed higher expression while $>1$ fold of IncRNA H19 had lower expression comparatively. It was also found that the IncRNA H19 expression had a negative correlation with miRNA-29a and miRNA-29b. This suggested that decreased IncRNA H19 expression showed to increased expression of miRNA-29a and miRNA-29b. In support of the present study, Hee et al. in 2007 and Karolina et al. in 2011 also revealed that the miRNA29 family was increased in several tissues, skeletal muscle, derived from diverse rat models of T2DM [34, 35]. It has also been revealed that miRNA-29a and miRNA-29b were up-regulated in T2DM patients' skeletal muscle tissue [36] study done by Zhang P et al in 2020, indicated that IncRNAs are important regulators of DM and are thus likely to serve as important diagnostic and therapeutic targets for this disorder [37]. Increased miRNA-29a expression was observed in T2DM and the present study provides support for the exploration of miR-29a antagonists as a potential therapeutic approach for T2DM treatment and the associated metabolic complications [38]. Huang $M$ et al revealed that IncRNA H19 could bind to miRNA-29a competitively following miRNA response elements (MREs) of miRNA-29a and affects the expression [15]. Zhong et al said that IncH19 sponge the miRNA-29b, [16] and Mengxin Lv et al also stated that $\mathrm{H} 19$ could directly bind to miR-29b and reduces its expression [17]. Over-expression of miR29a levels was positively associated with metabolic disorders and demonstrated to have a potential value as biomarkers in disease prognosis [39] as well as a study by Ying Zhi et al in 2018, stated that increased miRNAs miR-29b was observed in T2DM patients [40]. A study was done by Julie $\mathrm{M}$ et al in 2017 and revealed that miR-29 acts as an important regulator of insulin-stimulated glucose metabolism and showed relevance in type 2 diabetes [41].

\section{Conclusions}

The present study observed decreased expression of cell-free IncRNA H19 and increased expression of miRNA-29a as well as miRNA-29b in T2DM patients, Decreased expression was linked with hypertension, weight loss, and fatigue. Increased cell-free miRNA-29a expression was linked with hypertension, smoking, and alcoholism while miRNA-29b expression was linked with hypertension, weight loss, smoking, and alcoholism. This suggested that decreased expression of cell-free IncRNA H19, increased expression of miRNA-29a and miRNA-29b may be associated with the worseness of disease and involved in the pathogenesis of the disease. MiRNA-29a and miRNA-29b expression alteration observed to link with mainly smoking, alcoholism and this would be a factor for the severity of the disease. This data shed light on this gene interaction may be an essential factor for disease occurrence and worseness of disease. LncRNA H19, miRNA-29a, and miRNA-29b expression pattern information for the diagnosis/prognosis and management of T2DM cases. Further investigations are warranted to derive the difference in gene expression among T2DM cases.

\section{Declarations}

\section{Conflict of interest:}

None 


\section{Ethics approval and consent to participate:}

The study has been conducted only after the due clearance and approval from the Ethics committee of GKV (vide Proposal No. 14/08/2015/GKC/IEC/2015) has. As part of a mandatory standardized ethical norm, a written with signed informed consent was taken from the particular person before inclusion in the research work.

\section{Availability of data and materials:}

The datasets used and analysed during the present study would be available from the corresponding author.

\section{Funding Source:}

Deanship of Scientific Research at King Khalid University for funding this work through the General Research Project under grant number [G.R.P- 298].

\section{Acknowledgment:}

The authors extend their appreciation to the Deanship of Scientific Research at King Khalid University for funding this work through the General Research Project under grant number [G.R.P- 298]. We gratefully acknowledge the study subjects who were part of this study.

\section{References}

1. Rines AK, Sharabi K, Tavares CD, Puigserver P. Targeting hepatic glucose metabolism in the treatment of type 2 diabetes. Nat Rev Drug Discov. 2016; 15[11]:786-804.

2. Hatting M, Tavares CDJ, Sharabi K, et al. Insulin regulation of gluconeogenesis. Ann N Y Acad Sci. 2018; 1411[1]:21-35.

3. E. Bernstein, E. Birney, I. Dunham, et al, An integrated encyclopedia of DNA elements in the human genome, Nature, 489 [2012] 57-74.

4. R. Mercer, M.E. Dinger, J.S. Mattick, Long non-coding RNAs: insights into functions, Nat Rev Genet, 2009; 10 155-159.

5. C. Tsai, R.C. Spitale, H.Y. Chang, Long intergenic noncoding RNAs: new links in cancer progression, Cancer Res, 2011; 71 3-7.

6. Simion, V., Haemmig, S., and Feinberg, M. W. [2019]. LncRNAs in vascular biology and disease. Vascul. Pharmacol. 2018; 114, 145-156.

7. Gabory, A.; Jammes, H.; Dandolo, L. The H19 locus: Role of an imprinted non-coding RNA in growth and development. Bioessays 2010, 32, 473-480.

8. Gao, Y.; Wu, F.; Zhou, J.; et al. The H19/let-7 double-negative feedback loop contributes to glucose metabolism in muscle cells. Nucleic Acids Res. 2014, 42, 13799-13811. 
9. Bartel DP. MicroRNAs: genomics, biogenesis, mechanism, and function. Cell, [2004] 116:281-97.

10. Friedman RC, Farh KK, Burge CB, et al. Most mammalian mRNAs are conserved targets of microRNAs. Genome Res. [2009] 19:92-105.

11. Guay C, Roggli E, Nesca V, et al. Diabetes mellitus, a microRNA-related disease? Transl Res. [2011] 157:253-64.

12. Park SY, Jeong HJ, Yang WM, et al. Implications of microRNAs in the pathogenesis of diabetes. Arch Pharm Res. [2013] 36:154-66.

13. Kriegel AJ, Liu Y, Fang Y, Ding X, Liang M, et al. The miR-29 family: genomics, cell biology, and relevance to renal and cardiovascular injury. Physiol Genomics. 2012; 44:237-244.

14. Eyholzer M, Schmid S, Wilkens $L$, et al. The tumor-suppressive miR-29a/b1 cluster is regulated by CEBPA and blocked in human AML. Br J Cancer. 2010; 103:275-284.

15. Huang M, Zhong Z, Lv M, Shu J, Tian Q, Chen J, Comprehensive analysis of differentially expressed profiles of IncRNAs and circRNAs with associated co-expression and ceRNA networks in bladder carcinoma.

16. Min-Er Zhong, Yanyu Chen, Guannan Zhang, Lai Xu, Wei Ge, Bin Wu. LncRNA H19 regulates PI3KAkt signal pathway by functioning as a ceRNA and predicts poor prognosis in colorectal cancer: integrative analysis of dysregulated ncRNA-associated ceRNA network, Cancer Cell Int (2019) 19:148

17. Mengxin Lv, Zhenyu Zhong, Mengge Huang, Qiang Tian, Rong Jiang, Junxia Chen. IncRNA H19 Regulates Epithelial-Mesenchymal Transition and Metastasis of Bladder Cancer by miR-29b-3p as Competing Endogenous RNA.Biochim Biophys Acta Mol Cell Res. 2017 Oct;1864(10):1887-1899.

18. DeFronzo, R.A.; Abdul-Ghani, M. Type 2 diabetes can be prevented with early pharmacological intervention. Diabetes Care 2011, 34 [Suppl. 2], S202-S209.

19. Danaei G, Finucane MM, Lu Y, et al. National, regional, and global trends in fasting plasma glucose and diabetes prevalence since 1980: Systematic analysis of health examination surveys and epidemiological studies with 370 country-years and 2.7 million participants. Lancet 2011, 378, 3140.

20. Berends, L.M.; Ozanne, S.E. Early determinants of type-2 diabetes. Best Pract. Res. Clin. Endocrinol. Metab. 2012, 26, 569-580.

21. Gabory A, Jammes H, Dandolo L. The H19 locus: role of an imprinted non-coding RNA in growth and development. Bioessays. 2010;32[6]:473-480.

22. Gao Y, Wu F, Zhou J, et al. The H19/let-7 double-negative feedback loop contributes to glucose metabolism in muscle cells. Nucleic Acids Res. 2014, 42, 13799-13811.

23. Zhuo C, Jiang R, Lin X, et al. IncRNA H19 inhibits autophagy by epigenetically silencing of DIRAS3 in diabetic cardiomyopathy. Oncotarget [2017] 8:1429-37.

24. Moran VA, Perera RJ, Khalil AM. Emerging functional and mechanistic paradigms of mammalian long non-coding RNAs. Nucleic Acids Res [2012] 40:6391-400. 
25. Arnold N, Koppula P, Gul R, et al. Regulation of cardiac expression of the diabetic marker microRNA miR-29. PLoS One. 2014; 25:e103284.

26. Bagge A, Clausen TR, Larsen $S$, et al. MicroRNA-29a is up-regulated in beta-cells by glucose and decreases glucose-stimulated insulin secretion. Biochem Biophys Res Commun. 2012; 426:266-272.

27. Kong L, Zhu J, Han W, et al. Significance of serum microRNAs in prediabetes and newly diagnosed type 2 diabetes: a clinical study. Acta Diabetol. 2011; 48:61-69.

28. Long J, Wang $Y$, Wang W, et al. MicroRNA-29c is a signature microRNA under high glucose conditions that targets sprouty homolog 1 , and its in vivo knockdown prevents progression of diabetic nephropathy. J Biol Chem. 2011; 286:11837-11848.

29. Roggli E, Gattesco S, Caille D, et al. Changes in microRNA expression contribute to pancreatic betacell dysfunction in prediabetic NOD mice. Diabetes. 2012; 61:1742-1751.

30. Dahlmans D, Houzelle A, Jörgensen JA, et al. Evaluation of Muscle microRNA Expression in Relation to Human Peripheral Insulin Sensitivity: A Cross-Sectional Study in Metabolically Distinct Subject Groups.Front. Physiol. 2017; 8:711.

31. Massart J, Sjogren RJO, Lundell LS, et al. Altered miR-29 expression in type 2 diabetes influences glucose and lipid metabolism in skeletal muscle. Diabetes, 2017; 66:1807-18.

32. Zhou Y, Gu P, ShiW, et al.MicroRNA-29a induces insulin resistance by targeting PPARd in skeletal muscle cells. Int J Mol Med. 2016; 37:931-8.

33. Song H, Ding L, Zhang S, et al. MiR-29 family members interact with SPARC to regulate glucose metabolism.MiR-29 family members interact with SPARC to regulate glucose metabolism. BiochemBiophys Res Commun. 2018; 497:667-674.

34. He, A., Zhu, L., Gupta, N., et al. Overexpression of micro ribonucleic acid 29, highly up regulated in diabetic rats, leads to insulin resistance in 3T3-L1 adipocytes. Mol. Endocrinol. 2007; 21, 2785-2794.

35. Karolina, D. S., Armugam, A., Tavintharan, S., et al. MicroRNA 144 impairs insulin signaling by inhibiting the expression of insulin receptor substrate 1 in type 2 diabetes mellitus. PLoS ONE, 2011; 6:e22839.

36. Dahlmans D, Houzelle A, Jörgensen JA, et al. Evaluation of Muscle microRNA Expression in Relation to Human Peripheral Insulin Sensitivity: A Cross-Sectional Study in Metabolically Distinct Subject Groups. Front. Physiol. 2017; 8:711.

37. Zhang Pengyu, Yan Yan, Fu Xiying, Yang Maoguang, Li Mo, Cheng Yan, et al. The Differential Expression of Long Noncoding RNAs in Type 2 Diabetes Mellitus and Latent Autoimmune Diabetes in Adults, International Journal of Endocrinology Volume 2020, Article ID 9235329.

38. Peng Wu, Qianyi Wang, Cuilian Jiang, Chen Chen, Yun Liu, Yajun Chen et al, MicroRNA-29a is involved lipid metabolism dysfunction and insulin resistance in $\mathrm{C} 2 \mathrm{C} 12$ myotubes by targeting PPARס. Molecular Medicine Reports, 2018, 17: 8493-8501.

39. Xihua Lin, Cheng Luo, Dongjuan He, Eric Matro, Qilong Chen, Hong Li et al, Urinary miRNA-29a-3p levels are associated with metabolic parameters via regulation of IGF1 in patients with metabolic syndrome, BIOMEDICAL REPORTS, 2019, 10: 250-258. 
40. Ying-Zhi Liang, Jia-Jiang-Hui Li, Huan-Bo Xiao, Yan He, Ling Zhang, Yu-Xiang Yan, Identification of stress-related microRNA biomarkers in type 2 diabetes mellitus: A systematic review and metaanalysis, doi: 10.1111/1753-0407.12643.

41. Julie Massart, Rasmus J.O. Sjogren, Leonidas S. Lundell, Jonathan M. Mudry, Niclas Franck, Donal J. O'Gorman,3,4 Brendan Egan, Juleen R. Zierath, Anna Krook, Altered miR-29 Expression in Type 2 Diabetes Influences Glucose and Lipid Metabolism in Skeletal Muscle, Diabetes 2017;66:1807-1818.

\section{Figures}
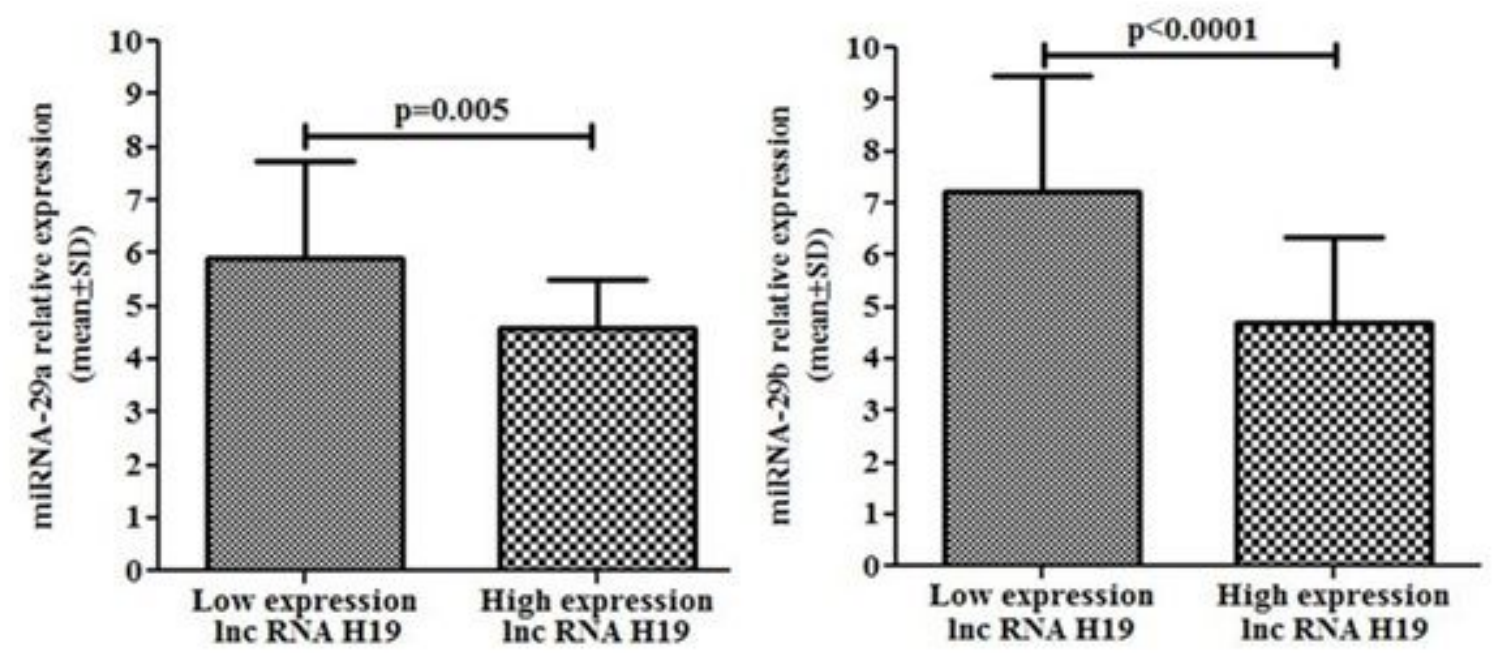

\section{Figure 1}

Association of IncRNA H19 expression with miRNA29a and miRNA29b expression.
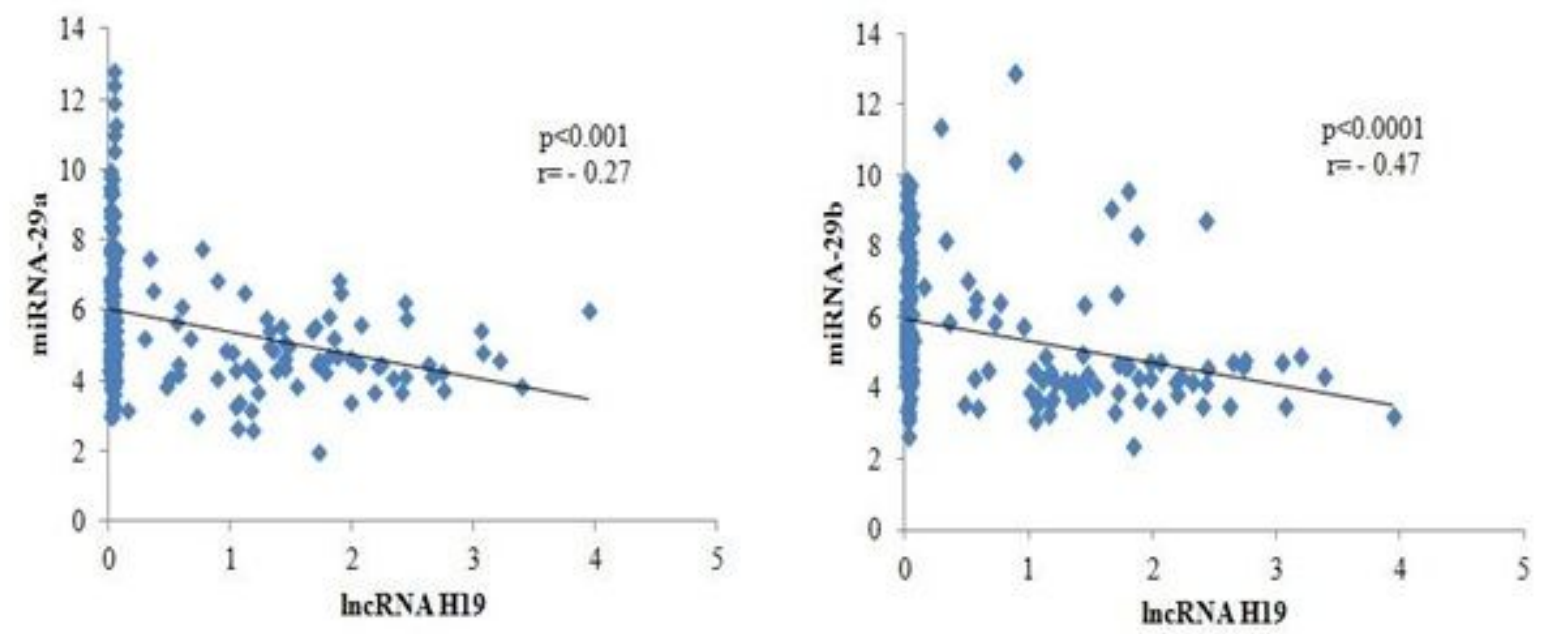

Figure 2

Correlation of IncRNA H19 with miRNA-29a and miRNA-29b 